\title{
BINP electron-positron facilities
}

D. Shwartz ${ }^{1,2, *}$ K. Astrelina ${ }^{1}$, V. Balakin ${ }^{1}$, A. Batrakov ${ }^{1}$, O. Belikov ${ }^{1}$, D. Berkaev ${ }^{1}$, D. Bolkhovitianov ${ }^{1}$, F. Emanov ${ }^{1}$, A. Frolov ${ }^{1}$, K. Gorchakov ${ }^{1}$, A. Kasaev ${ }^{1}$, A. Kirpotin ${ }^{1}$, I. Koop ${ }^{1,2}$, A. Krasnov ${ }^{1}$, N. Lebedev ${ }^{1}$, E. Levichev ${ }^{1}$, A. Lysenko ${ }^{1}$, Yu. Maltseva ${ }^{1}$, P. Martishkin ${ }^{1}$, O. Meshkov ${ }^{1,2}$, S. Motygin ${ }^{1}$, A. Murasev ${ }^{1}$, V. Muslivets ${ }^{1}$, A. Otboyev ${ }^{1}$, E. Perevedentsev ${ }^{1,2}$, P. Piminov ${ }^{1}$, V. Prosvetov ${ }^{1}$, Yu. Rogovsky ${ }^{1,2}, \quad$ A. Semenov ${ }^{1}$, A. Senchenko ${ }^{1,2}$, P. Shatunov ${ }^{1}$, Yu. Shatunov ${ }^{1,2}, \quad$ M. Timoshenko ${ }^{1}, \quad$ S. Vasiliev ${ }^{1}$, I. Zemlyansky ${ }^{1}$, Yu. Zharinov ${ }^{1}$, A. Zhuravlev ${ }^{1}$

${ }^{1}$ Budker Institute of Nuclear Physics, Novosibirsk, Russia

${ }^{2}$ Novosibirsk State University, Novosibirsk, Russia

Abstract. The present status of two operating BINP electron-positron colliders VEPP-2000 and VEPP-4M is given.

\section{Introduction}

Budker Institute of Nuclear Physics (BINP) has a long history of experiments at lepton colliders. Starting from pioneer electron-electron machine VEP-1 in early 1960s a number of electron-positron colliders served for several generations of particle detectors. Presently two operating colliders VEPP-4M and VEPP-2000 are joint via single new BINP Injection Complex (IC) and cover in common energy range from 0.16 to $5.5 \mathrm{GeV}$ per beam.

\section{Injection Complex}

IC is designed to supply BINP colliders with electron and positron beams. IC is a linacbased $e^{+} / e^{-}$beam source with Damping Ring (DR) and beam transfer lines to colliders. Linacs are based on $14 \mathrm{~S}$-band round disc-loaded waveguide accelerating structures which are fed with 4 SLAC 5045 klystrons. They are capable to reliably provide beams with energy about $400 \mathrm{MeV}$. Initially IC was designed to serve Charm-Tau factory, thus $700 \mathrm{MHz}(q=64) \mathrm{DR}$ RF cavity was used in order to provide damped bunch length about $1 \mathrm{~cm}$, suitable for further acceleration in S-band linac to experiment energy [1-3].

Since 2016 existing BINP colliders VEPP-2000 and VEPP-4M are using IC $\mathrm{e}^{+} / \mathrm{e}^{-}$beams in routine operation (see Fig. 1). Currently beams are accelerated to experiment energy by boosters, which can accept much longer bunch (up to $1 \mathrm{~m}$ full-length). In $2017 \mathrm{DR}$ RF cavity was replaced with $10.94 \mathrm{MHz}(q=1)$ one with maximum accelerating voltage of $10 \mathrm{kV}$ [3]. It allowed us to capture multi-bunch beam from pre-injector to damping ring with $40 \%$ increased efficiency. Currently achieved typical beam production parameters are shown in Table 1.

\footnotetext{
* Corresponding author: d.b.shwartz@inp.nsk.su
} 


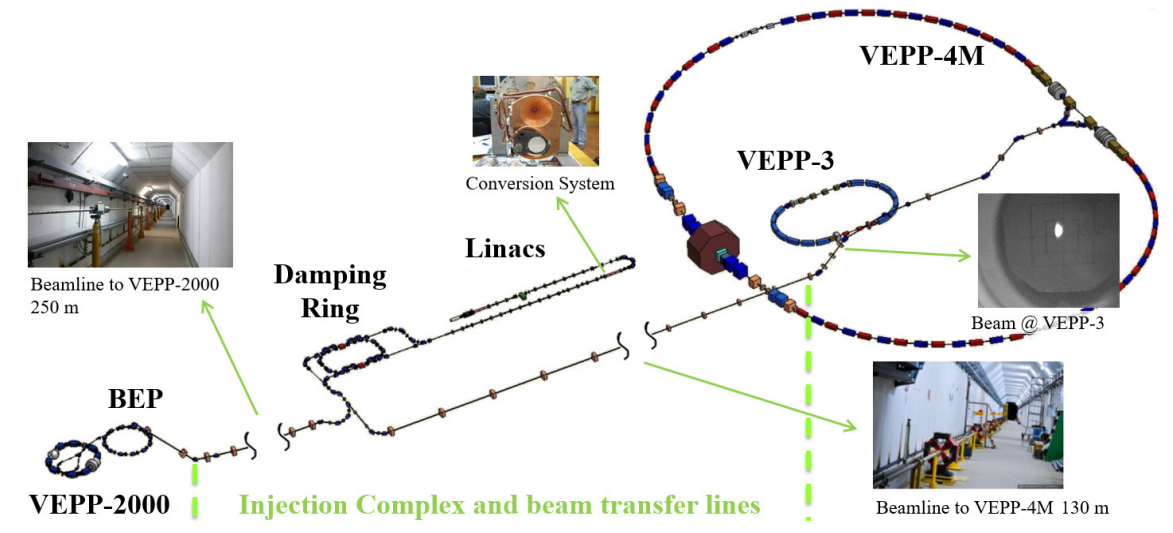

Fig. 1 IC serving two colliders.

Table 1. IC parameters.

\begin{tabular}{|l|l|}
\hline Beam energy & $395 \mathrm{MeV}$ \\
\hline Injection repetition rate & $12.5 \mathrm{~Hz}$ \\
\hline $\mathrm{e}^{-}$storage rate & Up to $1 \times 10^{10} /$ shot \\
\hline $\mathrm{e}^{+}$storage rate & $1 \times 10^{10} / \mathrm{s} @ 12 \mathrm{~Hz}$ injection rate \\
\hline
\end{tabular}

\section{VEPP-4M}

VEPP-4 is an electron-positron facility consisting of two storage rings VEPP-3 (up to $2 \mathrm{GeV}$ beam energy) and VEPP-4M (up to $\approx 5 \mathrm{GeV}$ ) [4]. The facility runs several research programs including high-energy physics in colliding mode [5], nuclear physics study with internal gas target [6], experiments with synchrotron radiation [7], external beam (electron, photon) test facility [8] and accelerator physics studies. Fig. 2 shows the facility schematically; Table 2 lists the main parameters.

Table 2. Parameters of VEPP-3 and VEPP 4M storage rings.

\begin{tabular}{|c|c|c|c|c|c|}
\hline Ring & VEPP-3 & \multicolumn{4}{|c|}{ VEPP-4M } \\
\hline Energy $(\mathrm{GeV})$ & $0.4-2.0$ & \multicolumn{4}{|c|}{$0.925-4.75(5.2)$} \\
\hline Circumference $(\mathrm{m})$ & 74.4 & \multicolumn{4}{|c|}{366} \\
\hline No of bunches & $2 \mathrm{e}^{-} / 2 \mathrm{e}^{+}$ & \multicolumn{4}{|c|}{$2 \mathrm{e}^{+} \times 2 \mathrm{e}^{-}\left(16 \mathrm{e}^{-}\right)$} \\
\hline Harmonic number & $2 / 18$ & \multicolumn{4}{|c|}{222} \\
\hline Betatron tunes, $\mathrm{h} / \mathrm{v}$ & $5.1 / 5.2$ & \multicolumn{4}{|c|}{$8.54 / 7.57$} \\
\hline Compaction factor & 0.076 & \multicolumn{4}{|c|}{0.0168} \\
\hline Coupling & 0.05 & \multicolumn{4}{|c|}{0.05} \\
\hline Beam Energy $(\mathrm{GeV})$ & 2.0 & 1.5 & 1.9 & 4.7 & 5.2 \\
\hline Bunch length $(\mathrm{cm})$ & 9 & \multicolumn{4}{|c|}{5} \\
\hline Emittance (nm) & 290 & 16 & 25 & 167 & 200 \\
\hline Energy Spread & 7.2 & 2.5 & 3.0 & 7.8 & 8.5 \\
\hline Bunch Current (mA) & 200 & 1.6 & 3.5 & 25 & 25 \\
\hline Luminosity $10^{30} \mathrm{~cm}^{-2} \cdot \mathrm{s}^{-1}$ & - & 0.9 & 3.3 & 44 & 25 \\
\hline
\end{tabular}




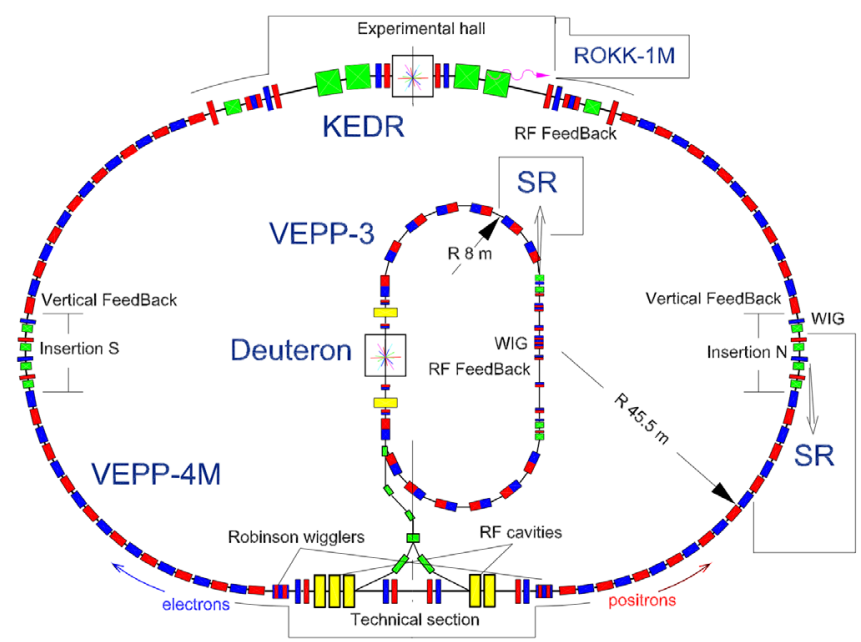

Fig. 2 VEPP-4 layout. Legend: KEDR is the universal superconducting magnet detector for HEP, deuteron is the nuclear physics facility at VEPP-3, SR are the synchrotron radiation halls, ROKK-1M is the external beam test hall.

We take $e^{+} e^{-}$beams from the BINP IC, transport them through $\sim 120 \mathrm{~m}$ long pulsed magnet transfer line and inject into VEPP-3 at energy of $390 \mathrm{MeV}$. The injection repetition rate is $1 \mathrm{~Hz}$. VEPP-3 (a) accelerates $e^{+} e^{-}$beams (alternatively) up to the maximum energy of $1.8 \mathrm{GeV}$ and delivers them into VEPP-4M, (b) polarizes $e^{+} e^{-}$beams for HEP in VEPP-4M, (c) performs separate experiments with SR and internal gas target.

The main research program of VEPP-4M is the colliding beams. In spite of low luminosity, the benefits of our collider are wide energy range (from 1 to $5 \mathrm{GeV}$ per beam), precise electron-positron tagging system for two-photon experiments and energy calibration by resonant depolarization with record accuracy of $10^{-6}$. Thanks to these advantages, we still stay afloat and make world level experiments. For instance, our mass measurements for $\mathrm{J} / \psi$ - and $\psi^{\prime}$-mesons are among seven most accurate particle mass measurements ever made.

In 2018, at VEPP-4M collider experimental program in low energy range (1-1.9 GeV per beam) was finished. The next run concentrates on:

- Hadronic cross-section measurement in the range of $2.3-3.5 \mathrm{GeV}\left(\sim 10 \mathrm{pb}^{-1}\right)$;

- Upsilon mesons study $\left(\sim 50 \mathrm{pb}^{-1}\right)$;

- Gamma-gamma physics $\left(\sim 200 \mathrm{pb}^{-1}\right)$.

The first stage of this program is the hadronic cross-section measurement in the beam energy range of 2.3-3.5 GeV. The first scan is finished and the results are shown in Fig. 3.

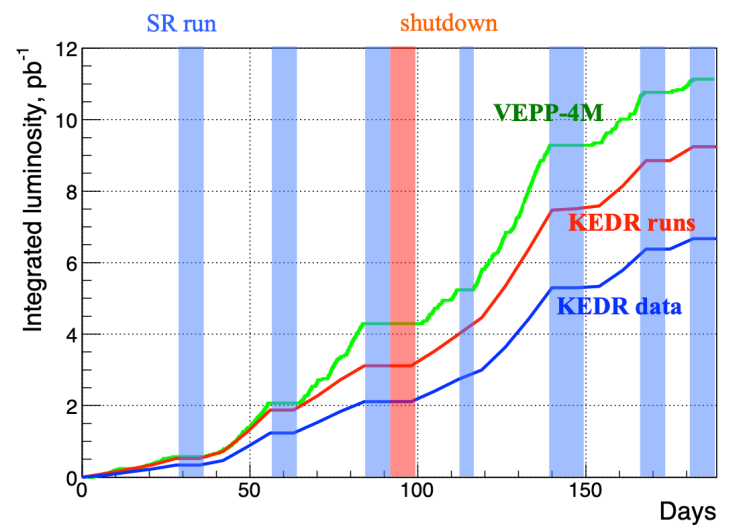

Fig. 3 The luminosity integral in first R-scan. 
To check the machine and detector ability, in May 2018 we have reached $\Upsilon(1 \mathrm{~S})$ at $4.75 \mathrm{GeV}$ and got the first luminosity. The main goal of the run was background study at high energy, and we found that the synchrotron radiation background is significantly higher than it was expected. To fix the background, new SR stoppers were developed and inserted in the vacuum tube around the KEDR detector.

Presently, the main problem for collider is a slow injection chain (mainly slow energy ramp up in VEPP-3 storage ring with non-laminated magnets). Many efforts are spent now to overcome the problem including development of new power supplies, control electronics and software, effective energy ramping algorithms, etc.

\section{VEPP-2000}

The VEPP-2000 collider [9-11] exploits the round beam concept (RBC) [12]. This approach, in addition to the straightforward geometrical gain factor in luminosity should yield the beam-beam limit enhancement. An axial symmetry of the disruptive nonlinear counter-beam force together with the $X-Y$ symmetry of the transfer matrix between the two IPs provide an additional integral of motion, namely, the longitudinal component of angular momentum $M_{z}=x^{\prime} y-x y^{\prime}$. Although the particles' dynamics remain strongly nonlinear due to beam-beam interaction, it becomes effectively one-dimensional. The reduction of degrees of freedom thins out the resonance grid and suppresses the diffusion rate resulting finally in a beam-beam limit enhancement [13].

Several demands upon the storage ring lattice suitable for the RBC appears:

1. Head-on collisions (zero crossing angle).

2. Small and equal $\beta$ functions at IP $\left(\beta_{x}^{*}=\beta_{y}^{*}\right)$.

3. Equal beam emittances $\left(\varepsilon_{x}=\varepsilon_{y}\right)$.

4. Equal fractional parts of betatron tunes $\left(v_{x}=v_{x}\right)$.

The first three requirements provide the axial symmetry of collisions while requirements (2) and (4) are needed for $X-Y$ symmetry preservation between the IPs.

\subsection{VEPP-2000 overview}

VEPP-2000 is a small $24 \mathrm{~m}$ in perimeter single-ring collider operating in one-by-one bunch regime in the energy range below $1 \mathrm{GeV}$ per beam. Its layout is presented in Fig. 4. Collider itself hosts two particle detectors [14, 15], Spherical Neutral Detector (SND) and Cryogenic Magnetic Detector (CMD-3), placed into dispersion-free low-beta straights. The final focusing (FF) is realized using superconducting $13 \mathrm{~T}$ solenoids. The main design collider parameters are listed in Table 3.

Table 3. VEPP- 2000 design parameters (at $\mathrm{E}=1 \mathrm{GeV}$ ).

\begin{tabular}{|l|l|}
\hline Parameter & Value \\
\hline Circumference, $C$ & $24.39 \mathrm{~m}$ \\
\hline Energy range, $E$ & $150-1000 \mathrm{MeV}$ \\
\hline Number of bunches & $1 \times 1$ \\
\hline Number of particles per bunch, $N$ & $1 \times 10^{11}$ \\
\hline Betatron functions at IP, $\beta_{x, y}^{*}$ & $8.5 \mathrm{~cm}$ \\
\hline Betatron tunes, $v_{x, y}$ & $4.1,2.1$ \\
\hline Beam emittance, $\varepsilon_{x, y}$ & $1.4 \times 10^{-7} \mathrm{~m} \mathrm{rad}$ \\
\hline Beam-beam parameters, $\xi_{x, z}$ & 0.1 \\
\hline Luminosity, $L$ & $1 \times 10^{32} \mathrm{~cm}^{-2} \mathrm{~s}^{-1}$ \\
\hline
\end{tabular}



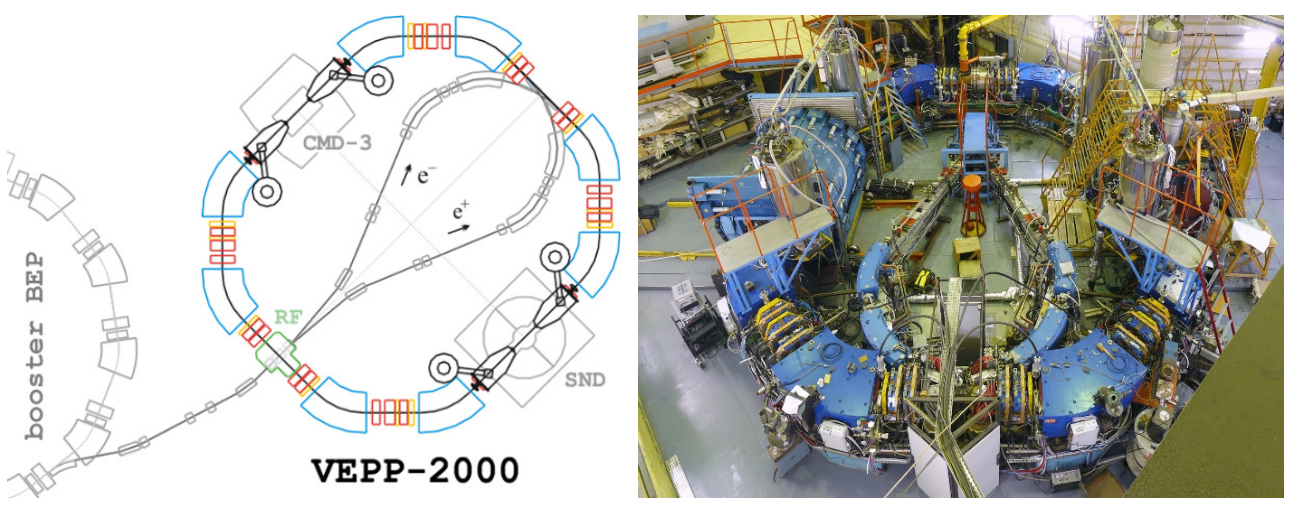

Fig. 4 VEPP-2000 layout and photo.

\subsection{Flip-flop effect and beam shaking}

The final beam-beam limit at VEPP-2000 corresponds to the onset of a flip-flop effect 16: the self-consistent situation when one of the beam sizes is blown-up while another beam size remains almost unperturbed. Observed in VEPP-2000 behavior is most likely caused by an interplay of beam-beam interaction and nonlinear lattice resonances.

The flip-flop threshold is sensitive to several tuning knobs, in particularly to $X-Y$ coupling and beta-functions misbalance at IP. In addition, the influence of bunch length on the threshold was observed.

In Fig. 5 (left) images from the online TV camera are presented for the cases of regular beams (a), blown-up electron beam (b) or positron beam (c). The corresponding coherent oscillations spectra are shown on the right. One can see in the spectra of a slightly kicked bunch that the shifted tune ( $\pi$-mode) sticks to the $1 / 5$ resonance in the case of a flip-flop.
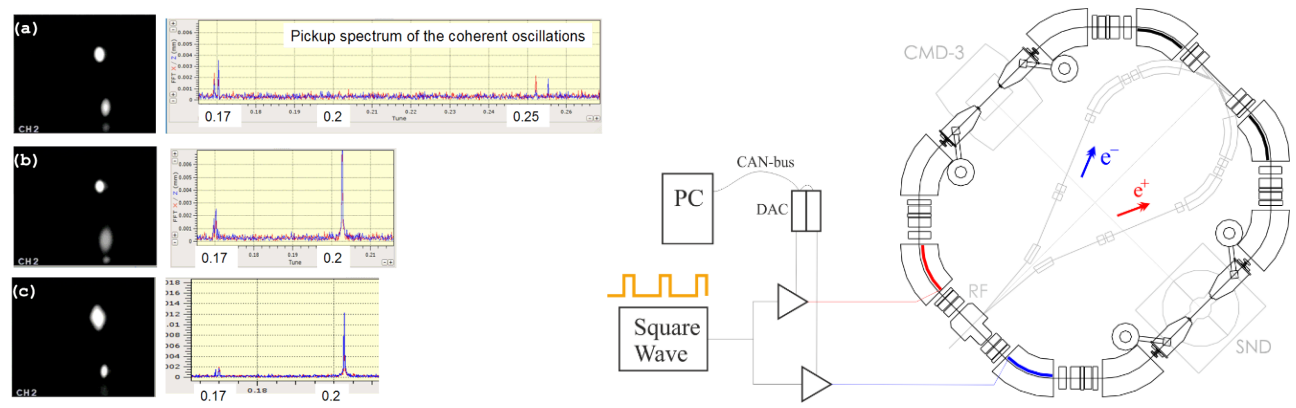

Fig. 5 Left: three flip-flop meta-stable states as visible in transverse profile and coherent beam-beam oscillations spectra. Right: the scheme of beam shaking.

While taking data at low energy range where the radiative emittance is small but significant beta-squeeze is not allowed due to the DA shrinking thus leaving mechanical aperture not fully used the natural desire appeared to increase the emittance. It allows to increase the beam current with fixed particles density, i.e. with fixed at the threshold beambeam parameter, and to increase luminosity linearly to beam intensity.

The idea was proposed to kick the beam weakly (in comparison to beam size) and frequently (in comparison to damping time). In the presence of strong nonlinear forces of colliding beam after the single kick the excited coherent oscillation decoheres very quickly thus increasing effective beam emittance.

The square wave generator was used to produce pulses of $\sim 300$ ns duration. Separated and amplified independently in two channels by software controlled amplificators pulses 
are applied to the additional kicker plates (not in use at low energies, see Fig. 5, bottom) in a running wave manner to affect only one beam per channel. In fact, in routine operation inevitably both beams are affected via beam-beam interaction.

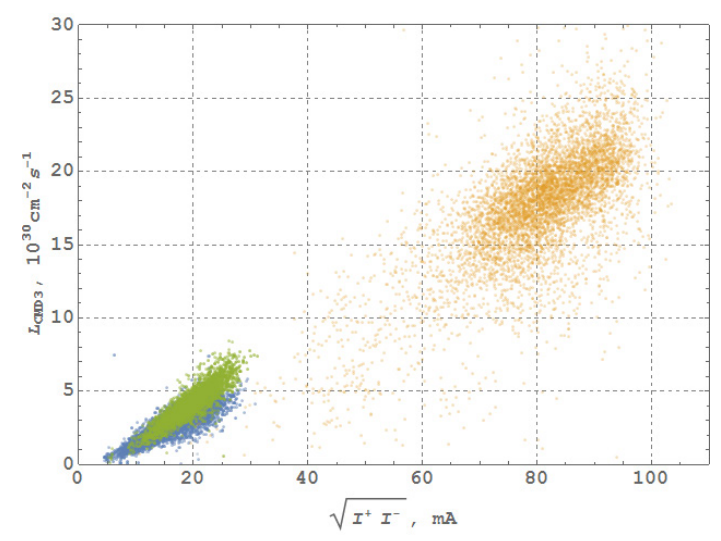

Fig. 6 The CMD-3 recorded luminosity as a function of beam currents. Blue and green dots correspond to machine performance in 2013 with short and long bunch correspondingly. Yellow dots correspond to 2018 .

The typical pulses parameters are the following: pulse duration $\sim 300 \mathrm{~ns}$ (3-4 turns), repetition rate $(50 \mu \mathrm{s})^{-1}$, pulse amplitude $50-100 \mathrm{~V}$ (depends on beam energy).

The beam shaking experimentally results in beams emittance growth. This growth depends on the controllable shaker parameters (pulse amplitude, pulse duration, repetition rate). The properly increased emittance prevents the flip-flop development during injection cycle: the "strong" beam can't shrink to unperturbed size when "weak" beam oscillates with large amplitudes. In addition, the beam lifetime is improved due to suppression of Touschek scattering with increasing emittance.

As a result of beam shaking technique implementation the beams intensities and luminosity at low energy range increased significantly. In Fig. 6 the luminosity is presented achieved in 2013 and in 2018 at the same given energy of $391 \mathrm{MeV}$.

\subsection{Data collection}

The 2016/17 run was the first data taking VEPP-2000 run with new injector [17-19]. It was dedicated to energy range from 640 to $1003.5 \mathrm{MeV}$ per beam. The design top energy was exceeded in order to achieve the mass of $D^{* 0}(2007)$. The run $2017 / 18$ was dedicated to the data collection at low beam energies: $274-600 \mathrm{MeV}$.

The achieved luminosity in comparison to 2010-2013 performance is shown in Fig. 7. In the middle energy range the achieved luminosity is well above all expectations. At the same time at top energy luminosity is lower than design value in a factor of two. 


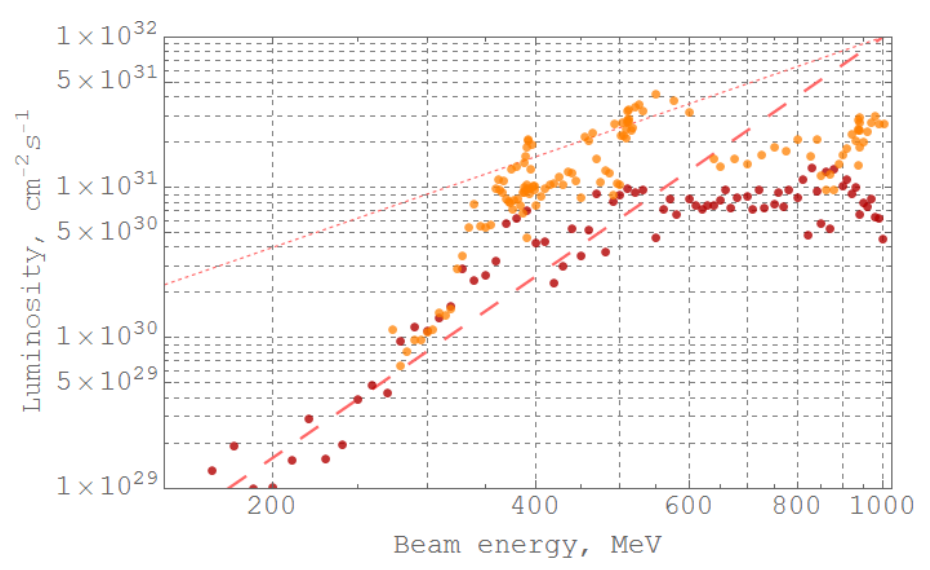

Fig. 7 CMD-3 recorded in 2010-2013 (crimson) and in 2017-2018 (orange) luminosity averaged over $10 \%$ of best runs. Pink lines show scaling laws with fixed and variable $\beta^{*}$.

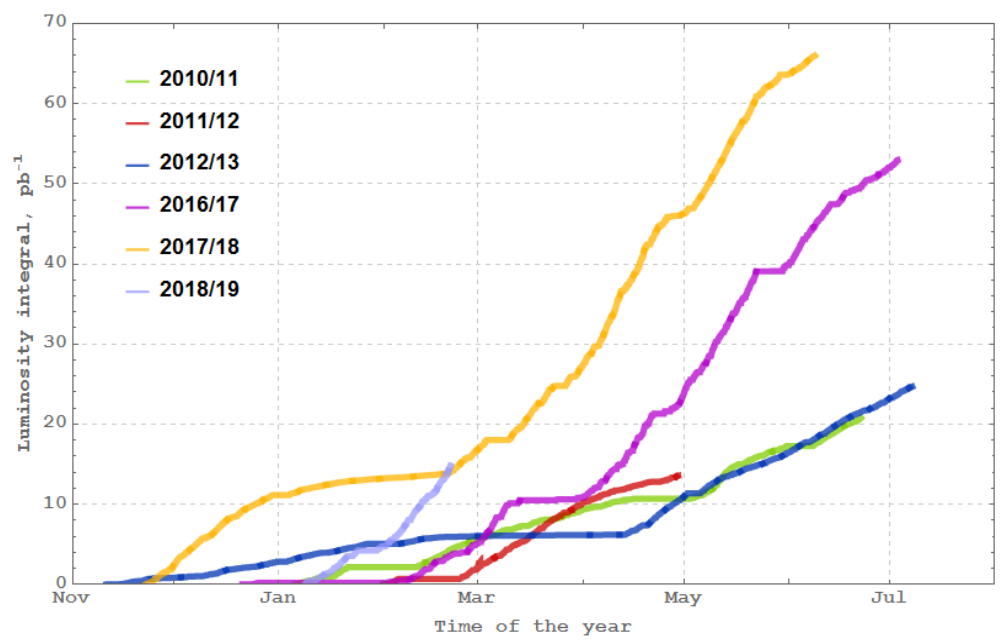

Fig. 8 CMD-3 recorded luminosity integral.

The Fig. 8 presents the integrated luminosity as compared for several operating years. One should beware of direct comparison of integrals due to luminosity dependence on energy. 2012/13 and 2017/18 runs were spent for data taking below $500 \mathrm{MeV}$ while the others were dedicated to higher energies.

\section{Future Facilities}

The main BINP future project for HEP is the Super Charm Tau factory [20]. It is an $e^{+} e^{-}$ collider with the beam energy range from 1 to $2.5 \mathrm{GeV}$ with extremely high luminosity $\left(\sim 10^{35} \mathrm{~cm}^{-2} \cdot \mathrm{s}^{-1}\right)$ and longitudinal polarization of electron beam at the IP. The facility can study the tau leptons, charmed particles and light quark spectroscopy in the unique manner.

Another ongoing project is small two-ring $e^{+} e^{-}$collider with very large crossing angle $\left(75^{\circ}\right)$ and beam energy of $408 \mathrm{MeV}$ to observe experimentally and study bound state of $\left(\mu^{+} \mu^{-}\right)$known as dimuonium with luminosity of $8 \times 10^{31} \mathrm{~cm}^{-2} \mathrm{~s}^{-1}[21]$. 


\section{Acknowledgements}

The work was done using the infrastructure of the Shared-Use Center "Complex VEPP-4-VEPP-2000".

\section{References}

1. K.V. Astrelina et al., "Production of Intense Positron Beams at VEPP-5 Injection Complex", Journal of Experimental and Theoretical Physics, Vol. 106, Issue 1, p. 77 (2008).

2. A. Starostenko et al., "Status of Injection Complex VEPP-5: Machine Commissioning and First Experience of Positron Storage", in Proc. IPAC'14, Dresden, Germany p. 538 (2014).

3. Yu. Maltseva et al., "VEPP-5 Injection Complex: New Possibilities for BINP Electron-Positron Colliders", in Proc. IPAC'18, Vancouver, Canada, p.371 (2018).

4. E.B. Levichev, "Status and upgrade of the VEPP-4 storage-ring facility", Phys. Part. Nucl. Lett. 13 no.7, p.876 (2016).

5. P. Piminov, "Status of the electron-positron collider VEPP-4", in Proc. IPAC'17, Copenhagen, Denmark, p.2985 (2017).

6. D.M. Nikolenko, "Experiments with internal targets at the VEPP-3 electron storage ring", Phys. Atom. Nucl., 73, 8, p.1322 (2010).

7. P. Piminov et al., "Synchrotron radiation research and application at VEPP-4", Physics Procedia 84, 19, p.26 (2016).

8. V.S. Bobrovnikov et al, "Extracted electron and gamma beams in BINP", JINST 9 C08022 (2014).

9. Yu.M. Shatunov et al., "Project of a New Electron-Positron Collider VEPP-2000", in Proc. EPAC'00, Vienna, Austria, p. 439 (2000).

10. D. E. Berkaev et al., "The VEPP-2000 electron-positron collider: First experiments", J. Exp. Theor. Phys., Vol.113, no.2, p.213 (2011).

11. Yu.M. Shatunov et al., "Recommissioning the Modernized VEPP-2000 Electron-Positron Collider", Phys. Part. Nucl. Lett. 15, no.7, p.749 (2018).

12. V.V. Danilov et al., "The Concept of Round Colliding Beams", in Proc. EPAC'96, Sitges, Spain, p. 1149 (1996).

13. K. Ohmi, K. Oide and E.A. Perevedentsev, "The beam-beam limit and the degree of freedom", in Proc. EPAC'06, Edinburgh, Scotland, p.616 (2006).

14. M. Achasov et al., "Recent results from SND detector at VEPP-2000 collider", these proceedings.

15. F. Ignatov et al., "Recent results from CMD-3", these proceedings.

16. D. Shwartz et al., "Recent Beam-Beam Effects at VEPP-2000 and VEPP-4M", in Proc. ICFA Mini-Workshop on Beam-Beam Effects in Hadron Colliders (BB2013), Geneva, Switzerland, CERN-2014-004, p. 43 (2013).

17. P.Yu. Shatunov et al., "Status and perspectives of the VEPP-2000", Phys. Part. Nucl. Lett. 13, p. 995 (2016).

18. D. Berkaev et al., "Commissioning of Upgraded VEPP-2000 Injection Chain", in Proc. IPAC'16, Busan, Korea, p. 3811 (2016).

19. D. Shwartz et al., "Recomissioning and Perspectives of VEPP-2000 e+e- Collider", PoS ICHEP2016, p.054 (2016).

20. I. Logashenko et al., "Super Charm-Tau project at Novosibirsk", these proceedings.

21. A. Bogomyagkov et al., "Low-energy electron-positron collider to search and study (mu+ mu- ) bound state", these proceedings. 